\title{
The evaluation of mechanical properties of animal tendons after corticosteroid therapy
}

\author{
Grażyna Olchowik ${ }^{1}$, Elżbieta Siek ${ }^{2}$, Monika Tomaszewska ${ }^{3}$, Marek Tomaszewski ${ }^{4}$ \\ ${ }^{1}$ Departament of Biophysics, Medical University of Lublin, Poland \\ ${ }^{2}$ First Department of Radiology, Medical University of Lublin, Poland \\ ${ }^{3}$ Department of Human Anatomy, Medical University of Lublin, Poland
}

\begin{abstract}
The purpose of the study was to examine the effect of systemic glucocorticosteroids administration on mechanical properties of animal tendons. Twenty female Wistar rats were divided into two groups and those that formed experimental group were subjected activity of hydrocortisone given to the peritoneum. Mechanical tests were performed. Hydrocortisone administration caused reduction of force value and the increase of strain, also the value of elasticity module was significantly smaller comparing to the control group. The results of biomechanical tests suggested that glucocorticoids produce less stiffness tendon, which fails in maximum point of load. This study revealed that systemic hydrocortisone administration changes significantly mechanical properties of tendons, which may cause frequent failure of the tendon.
\end{abstract}

Key words: Hydrocortisone - Mechanical properties - Achilles tendon - Systemic administration - Peritoneum

\section{Introduction}

Glucocorticosteroids are 21-carbon steroids that demonstrate numerous biological activities. Their systemic administration is widespread in the treatment of various pathological conditions like adrenal cortex hormone deficiency, allergies, systemic lupus erythematosus, rheumatoid arthritis, asthma, ulcerative colitis, Crohn's disease and many other rheumatoid, haematological or dermatological diseases. They are also administered locally as articular, periarticular injections in diseases or for trauma of the costo-articular-muscular system. Regardless of the administration route they have a beneficial antiinflammatory and analgesic impact, and thank to their immunosuppressive action they may be used in the treatment of patients after solid organ transplantation. However, except for the beneficial effect, glucocorticosteroids cause numerous and serious adverse effects. They cause increases in the susceptibility to infections, thrombus formation, diabetes, and they prolong wound healing. The literature also

Correspondence: G. Olchowik, Dept. of Biophysics, Medical University of Lublin, Jaczewskiego 6, 20-090 Lublin, Poland;

e-mail: grazyna.olchowik@am.lublin.pl describes the occurrence of iatrogenic Cushing syndrome, peptic and duodenal ulcers, cataracts, glaucoma, myopathy, osteoporosis, pathological breaks and aseptic bone necrosis. Reports exist of spontaneous tendon tears in patients having used the glucocorticosteroid treatment [1-5].

The literature contains inconsistent reports concerning the influence of glucocorticosteroid administration on the tendons. Various experimental studies describe a disadvantageous influence by glucocorticosteroids when given locally on the properties of the mechanical tendons, as manifested in changes to the biomechanical properties [6-9]. However, the authors of another paper did not state any significant differences in the mechanical properties of tendons between the control group and the group subjected to local glucocorticosteroid action [10]. Despite the belief in the disadvantageous influence of systematically administered glucocorticosteroids on tendons, except for reports of spontaneous Achilles tendon tears in numerous clinical cases, there is a lack in the literature of well-documented reports concerning studies on the biochemical parameters of tendons on animal models.

This paper presents the studies to evaluate the influence of the hydrocortisone administered to the peritoneum on the tendons of experimental animals. 


\section{Materials and Methods}

Studies were conducted on mature female rats of the Wistar breed, of 12 weeks of age, complying with current rules on animal care and use for experimental studies. Studies were conducted under an approval of the Bioethical Committee of the Medical University of Lublin. Animals came from a licensed breeding centre.

Before initiation of the principle studies, the animals were subjected to a 14-day adaptation process for the experimental conditions. Animals were fed using a standard diet. They had constant physical conditions preserving a natural lighting cycle (day/night) and free access to fodder and water, according to the recommendations for the animal breed and use for experimental purposes. Before initiation of the principle studies, animals were randomly assigned to two groups (experimental and control), with 10 rats in each group.

The average initial weight of the animals did not differ significantly between the groups used for experiments (Table 1). Following experiment finalization, no statistically significant differences were observed in the animal's weight.

\section{Experiment description}

Animals from the experimental group (HC) were given hydrocortisonum hemisuccinatum (Polfa, Jelenia Góra) to the peritoneum for 10 weeks in single doses of 10 $\mathrm{mg} / \mathrm{kg}$ per day. Animals from the control group (K) were given saline $(0.9 \% \mathrm{NaCl})$ to the peritoneum in doses of $0.25 \mathrm{ml} / 100 \mathrm{~g}$ body weight. On the $21^{\text {st }}$ day after the final hydrocortisone administration, the animals were killed through decapitation. The Achilles tendons along with the attachments were collected for the studies. Samples for the biomechanical tests were stored in saline $(0.9 \% \mathrm{NaCl})$ at the temperature of $+4^{\circ} \mathrm{C}$. The evaluation of the changes occurring in the tendons was conducted based on the resistance tests.

The mechanical tests were performed using a testing machine (Lloyd, type LRX). The clamping system was composed of metal blocks with serrated grips with smooth-surface teeth. The tendon-calcaneus complex was mounted vertically with calcaneus at the upper clamp and the muscle tendon junction at the lower clamp. The distance between the two attachment grips at a load of $0.1 \mathrm{~N}$ was considered as the reference length. The diameter of the middle part of the tendon was measured with calipers. Each tendon was stretched to failure at a rate of $10 \mathrm{~mm} / \mathrm{min}$ without any preconditioning and the load-deflection curve was recorded. The following parameters were analyzed: the highest registered force (load max), the force at proportional limit (load $\mathrm{H}$ ), strain at maximum load (strain max), strain at proportional limit (strain $\mathrm{H}$ ) and stiffness of the tendon (stiffness max, stiffness average). Maximum stiffness was calculated as the slope of the linear portion of the load-extension plot after the characteristic nonlinear "toe" response and before the first sign of deflection from proportionality. Given the low elongation rate resulting in a large toe region, "the average stiffness" was also measured for $0.2 \mathrm{~mm}$ increments of extension from the start to the limit of
Table 1. Average body weight of the rats in the K-control group, HC-subjected to the hydrocortisone action.

\begin{tabular}{|l|c|c|c|}
\hline Group & $\begin{array}{c}\text { Baseline body } \\
\text { weight } \\
(\mathrm{g})\end{array}$ & $\begin{array}{c}\text { Final body } \\
\text { weight } \\
(\mathrm{g})\end{array}$ & $\begin{array}{c}\text { Body weight } \\
\text { gain } \\
(\mathrm{g})\end{array}$ \\
\hline $\mathrm{K}$ & $310.0 \pm 7.9$ & $338.8 \pm 7.7$ & $28.8 \pm 3.6$ \\
\hline IIC & $313.4 \pm 6.4$ & $347.1 \pm 6.4$ & $33.7 \pm 3.2$ \\
\hline
\end{tabular}

Table 2. Results significance levels of the differences between groups (C - control group, $\mathrm{HC}$ - hydrocortisone administration group).

\begin{tabular}{|l|c|}
\hline \multicolumn{1}{|c|}{ Parameters } & IIC vs. C t-test \\
\hline Load max & Ns \\
\hline Load H & 0.033 \\
\hline Strain max & 0.002 \\
\hline Strain H & 0.005 \\
\hline Stiffness max & 0.001 \\
\hline Stiffness average & $<0.05$ \\
\hline Young's modulus & $<0.01$ \\
\hline
\end{tabular}

proportionality. Strain values were expressed by considering the elongation at a given point and the reference length. During measurements tendons were kept wet in saline solution.

The mean values and standard deviations were determined based on the results from all animal groups. The significance analysis of the differences between groups was performed using the t-test. Significance between groups was accepted at level of $\mathrm{p}<0.05$.

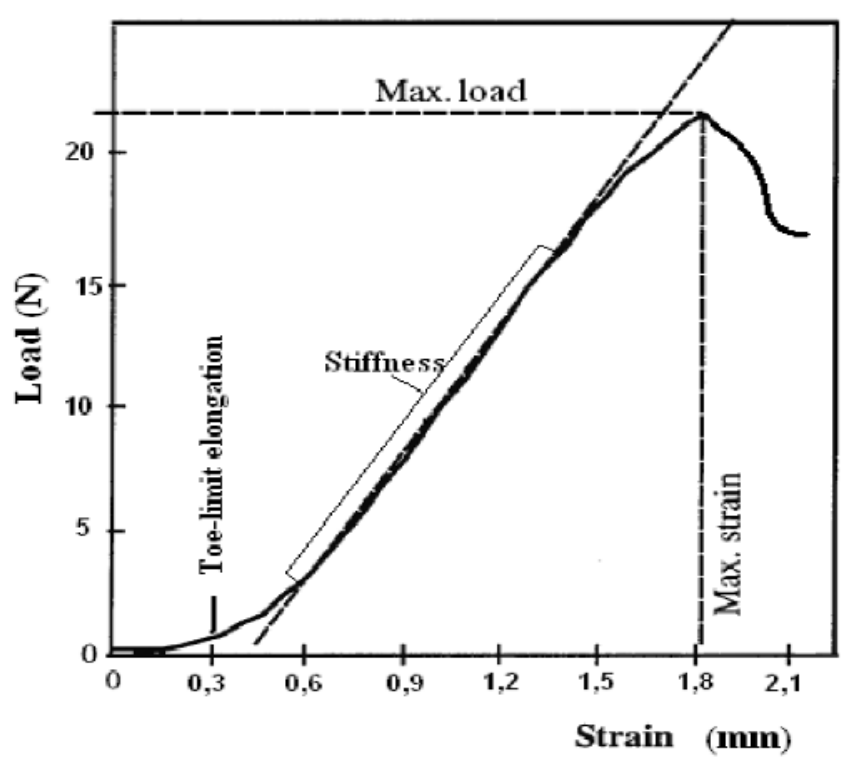

Fig. 1. Typical load-elongation data with definitions of biomechanical properties for tendon of rats. 


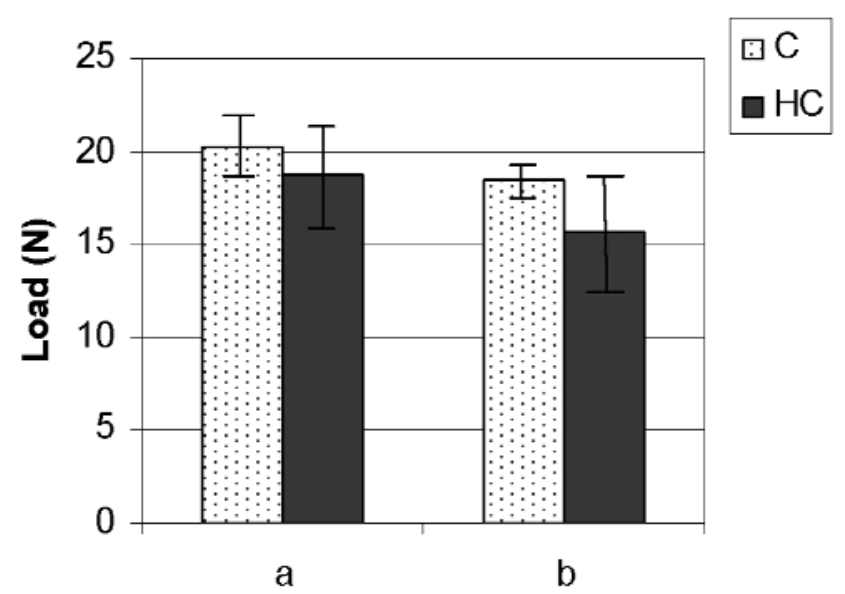

Fig. 2. The load value at the maximum load point (a) and at the proportional limit (b) in two groups of animals: control group (C) and group were treated hydrocortisone $(\mathrm{HC})$.

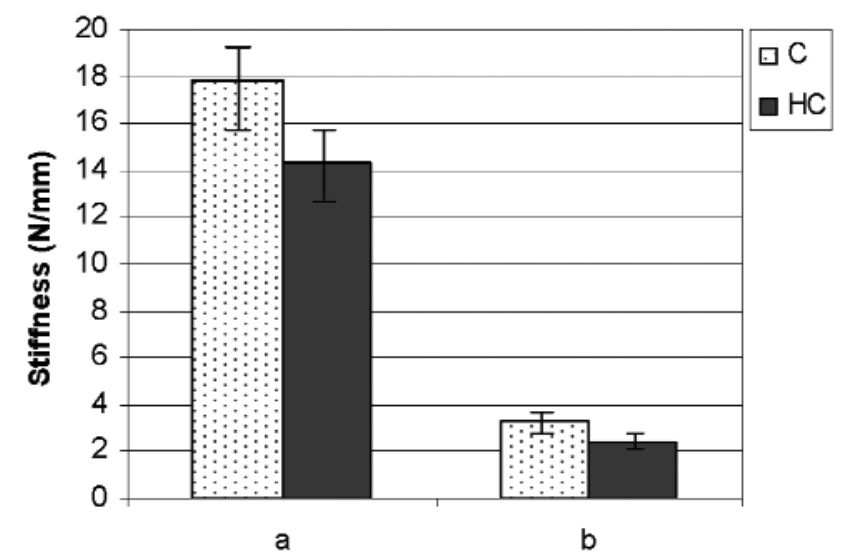

Fig. 4. The stiffness value at the maximum load point (a) and the average stiffness (b) in two groups of animals: control group (C) and group were treated hydrocortisone (HC).

\section{Results}

The studies and calculation results from the determined biomechanical parameters are shown in Fig. 1. Significance levels of the differences between groups are shown in the Table 2.

The results of the biomechanical parameters determined at the point of maximum load and at the strain limit show the disadvantageous effect of hydrocortisone on the tendons. From the results given in Fig. 2, for the group of rats subjected to hydrocortisone, load values at the maximum load point were $7.9 \%$ lower compared to the animals from the control group $(\mathrm{p}=0.06)$. However, the strain value to the point of maximum load was $21.6 \%$ greater compared to the control group, with a significance level $\mathrm{p}=0.001$ (Fig. 3). Tendons from animals of group $\mathrm{HC}$ also showed $35 \%(\mathrm{p}=0.001)$ less stiffness compared to the control animals (Fig. 4).

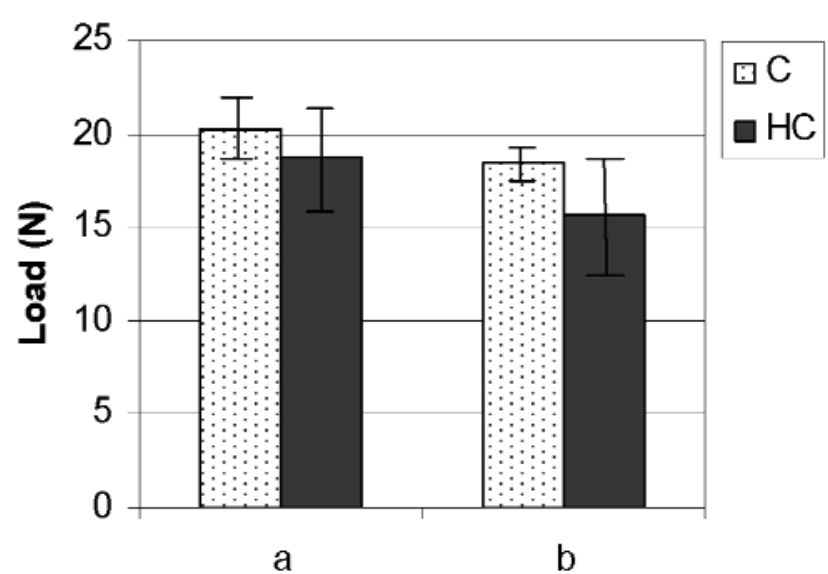

Fig. 3. The strain value at the maximum load point (a) and at the proportional limit (b) in two groups of animals: control group (C) and group were treated hydrocortisone $(\mathrm{HC})$.

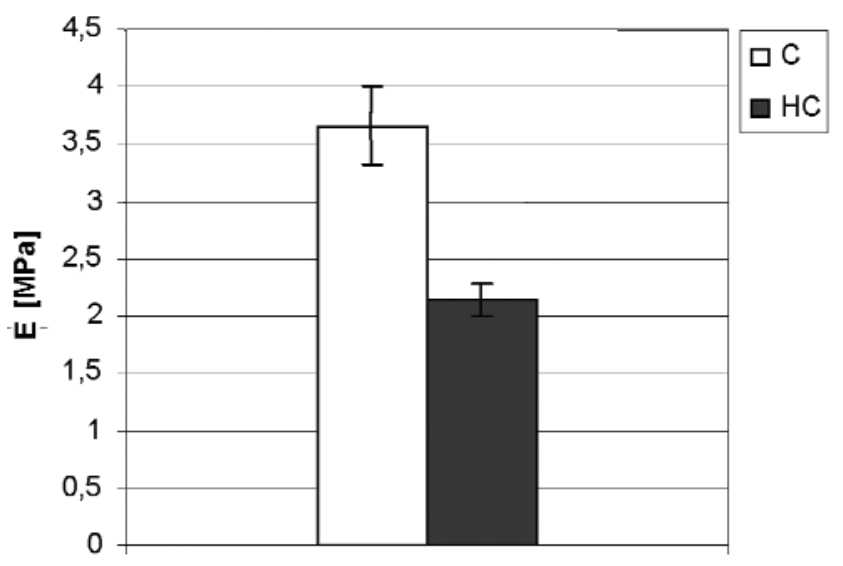

Fig. 5. The value of elasticity (E) in two groups of animals: control group (C) and group were treated hydrocortisone (HC).

During daily physical activity of the tendons, strains occur that do not exceed the range of the elastic deformation. Deformations within that range are reversible i.e. relaxing upon mechanical load removal. Parameters determined on the elasticity limit are of important practical significance, for above that point the tendon undergoes a permanent deformation and loses its function.

Performed mechanical tests showed significant statistical differences both in the tendon load value and in deformities on the limit of the elasticity between the animal groups. In the group of animals given hydrocortisone, strain was $37.9 \%$ greater, and load value was $15.3 \%$ smaller.

The indicator determining structure susceptibility to deformities is the elasticity module. The value of elasticity in animals subjected to the steroid therapy was $41.5 \%$ smaller compared to the control group (Fig. 5). Statistically significant $(\mathrm{p}<0.01)$ reductions in 
the value of elasticity module are probably a consequence of structural changes to the collagen fibres. Reductions in their number and quantitative and qualitative changes in the connections between them cause reductions in tendon elasticity, and hence toe-limit elongation segment shortening. The toe-limit elongation is the difference between the zero-force intercept of the linear portion of the force-elongation curve and the elongation reference point. The curve course in the "toe" segment is also influenced by animal mass.

\section{Discussion}

The results of the mechanical tests show that long-term hydrocortisone administration leads to disadvantageous structural changes in tendons and reductions in load values causing them to tear.

The existing literature confirms the disadvantageous effect of glucocorticosteroids on tendon biomechanical properties.

Hugate et al. [6] conducted a study on tendon biomechanics, giving glucocorticosteroid to rabbits in the form of an injection to the joint and to the bursa. They observed a reduction in elasticity, total resistance and tearing force in the tendons compared to the control group. The same experiment also showed that the medicine administration site is independent of the effect on the tendons.

Miles et al. [11] studied the dependence of anabolic steroid administration and efficiency training. They stated that steroids cause the formation of more rigid tendons, via increases in collagen fibre numbers and defective connections between them, which may lead to the weakening of the tendons and the increase in predisposition to tearing, even with smaller elongations. Changes in the biomechanical parameters of the tendons are probably the result of structural changes to the collagen fibre. Cutroneo and Counts suggest that treatment with glucocorticoids is involved in the impairment of collagen biosynthesis. Their data indicates that administration of these drugs results in a decrease of prolyl hydroxylase activity and it causes the decrease of hydroxyproline formation [12].

Toricelli et al. [9] showed significant changes in tenocytes vitality and the proliferation (determining proper tendons function) and reduction in collage type I and proteoglycans production (responsible for tendon resistance and elasticity) in animals subjected to systemic corticosteroid action. They stated that such a method of corticosteroid administration in terms of its action at the cellular level decreases tendon resistance and increases the incidence of trauma.

However, Shrier et al. [10] suggest that reductions in tendon tear force following local corticosteroid administration may be caused by numerous injections performed over many weeks and the accumulation of micro-trauma in that area and the occurrence of degenerative changes. Similar results got Wei et al. [13]. They came to a conclusion that a successive dose of steroid in uninjured tendons initiates a short-term response equivalent to that of structural injury.

The literature also contains clinical reports concerning the relationship between chronic steroid use and spontaneous tendon tearing. Isenberg et al. [14] presented a case of footballer who had taken anabolic steroids for three years and because of that later needed surgery for rupture of the patellar tendon and of both Achilles tendons. Newnham and co-authors [5] in their study presented the results of 10 patients treated with prednisolone in doses of $5-10 \mathrm{mg}$ and inhaled corticosteroids due to asthma or chronic obturatory pulmonary disease, where Achilles tendon tearing was detected during the therapy. They also observed a relationship between spontaneous tendon tear occurrence and the taking of oral or inhaled steroids, which very rarely occurred in patients with diagnosed obturatory pulmonary disease not treated with steroids. A similar case was also described by Kurhana et al. [3] for a patient with chronic obturatory pulmonary disease, where a bilateral Achilles tendon tear occurred during the treatment.

Some authors [3,5] warn, however, that spontaneous Achilles tendon tearing in such patients may be caused by degenerative changes occurring in this tissue in elderly patients, and medicine administration may only intensify the trauma occurrence frequency.

For patients with systemic diseases [4], dialysed patients [2] and after solid organs transplantation [15], the literature describes cases of tendon tearing and trauma incidence as being a rare complication of the steroid therapy. However, those researchers emphasised that additional ischemic and inflammatory changes influencing the tendon, in the course of the primary disease, should not be excluded.

On the background of such divergent opinions, the results of the conducted studies indicate that hydrocortisone administration to the peritoneum has a systemic effect and significantly changes the elasticity, and as a result changes the resistance to tear of the tendons.

\section{References}

[1] Fitzgerald BT, Hofmeister EP, Fan RA, Thompson MA. Delayed flexor digitorum superficialis and profundus ruptures in a trigger finger after a steroid injection: A case report. $J$ Hand Surg. 2005;30(3):479-482.

[2] Jones N, Kjellstrand CM. Spontaneous tendon rupture in patients on chronic dialysis. Am. J. Kidney Dis. 1996;28:861866.

[ 3] Khurana R, Torzillo PJ, Horsley M, Mahoney J. Spontaneous bilateral rupture of the Achilles tendon in a patient with chronic obstructive pulmonary disease. Respirology. 2002; 7(2):161-163.

[ 4] Kissel C, Sundaresson A, Unrae B. Spontaneous Achilles tendon rupture in a patient with SLE. J. Foot Surg. 1989;30: 918. 
[5] Newnham DM, Douglas JG, Legge JS, Friend JA. Achilles tendon rupture: an underrated complication of corticosteroid treatment. Thorax. 1991;46:853-854.

[ 6] Hugate R, Pennypacker J, Saunders M, Juliano P. The effects of intratendinous and retrocalcaneal intrabursal injections of corticosteroid on the biomechanical properties of rabbit Achilles tendons. J Bone Joint Surg Am. 2004;86: 794-801.

[7] McWhorter JW, Francis RS, Heckmann RA. Influence of local steroid injections on traumatized tendon properties. A biomechanical and histological study. Am J Sport Med. 1991; 19:435-439.

[ 8] Tatari H, Kosay C, Baran O, Ozcan O, Ozer E. Deleterious effects of local corticosteroid injections on the Achilles tendon of rats. Arch Orthop Trauma Surg. 2001;121(6):333-337.

[ 9] Torricelli P, Fini M, Giavaresi G, Carpi A, Nicolini A, Giardino R. Effects of systemic glucocorticoid administration on tenocytes. Biomed Pharmacother. 2006;60:380-385.

[10] Shrier I, Matheson GO, Kohl HW. Achilles tendinitis: are corticosteroids injections useful or harmful? Clin J Sport Med. 1996;6:245-250.
[11] Miles JW, Grana WA, Egle D, Min KW. The effect of anabolic steroids on the biomechanical and histological properties of rat tendon. J Bone Joint Surg Am. 1992;74-A (3):411-422.

[12] Cutroneo K.R., Counts D.F. Anti-inflammatory steroids and collagen metabolism: glucocorticoid-mediated alterations of prolyl hydroxylase activity and collagen synthesis. Mol Pharmacol. 1975;11(5):632-639.

[13] Wei AS, Callaci JJ, Juknelis D et al. The effect of corticosteroid on collagen expression in injured rotator cuff tendon. J Bone Joint Surg Am. 2006;88(6):1331-1338

[14] Isenberg J., Prokop A., Skouras E. Successive ruptures of patellar and Achilles tendons: Anabolic steroids in competitive sports. Unfallchirurg. 2008;111(1):46-49.

[15] Pirson Y, Ghysen J, Squifflet JP, Alexandre GPJ, van Ypersele de Strihou C. Multiple spontaneous rupture of tendons in renal transplant patients. BMJ. 1984;288:1010.

Submitted: 25 October 2007 Accepted after reviews: 14 February 2008 